\title{
Automated workflow-based exploitation of pathway databases provides new insights into genetic associations of metabolite profiles
}

Harish Dharuri', Peter Henneman ${ }^{2}$, Ayse Demirkan 1,3, Jan Bert van Klinken ${ }^{1}$, Dennis Owen Mook-Kanamori, 2,4, Rui Wang-Sattler ${ }^{5}$, Christian Gieger ${ }^{6}$, Jerzy Adamski ${ }^{7,8}$, Kristina Hettne ${ }^{1}$, Marco Roos ${ }^{1}$, Karsten Suhre ${ }^{4,9}$, Cornelia M Van Duijn ${ }^{3}$, EUROSPAN consortia, Ko Willems van Dijk ${ }^{1,10}$ and Peter AC 't Hoen ${ }^{1 *}$

\begin{abstract}
Background: Genome-wide association studies (GWAS) have identified many common single nucleotide polymorphisms (SNPs) that associate with clinical phenotypes, but these SNPs usually explain just a small part of the heritability and have relatively modest effect sizes. In contrast, SNPs that associate with metabolite levels generally explain a higher percentage of the genetic variation and demonstrate larger effect sizes. Still, the discovery of SNPs associated with metabolite levels is challenging since testing all metabolites measured in typical metabolomics studies with all SNPs comes with a severe multiple testing penalty. We have developed an automated workflow approach that utilizes prior knowledge of biochemical pathways present in databases like KEGG and BioCyc to generate a smaller SNP set relevant to the metabolite. This paper explores the opportunities and challenges in the analysis of GWAS of metabolomic phenotypes and provides novel insights into the genetic basis of metabolic variation through the re-analysis of published GWAS datasets.

Results: Re-analysis of the published GWAS dataset from Illig et al. (Nature Genetics, 2010) using a pathway-based workflow (http://www.myexperiment.org/packs/319.html), confirmed previously identified hits and identified a new locus of human metabolic individuality, associating Aldehyde dehydrogenase family1 L1 (ALDH1L1) with serine/ glycine ratios in blood. Replication in an independent GWAS dataset of phospholipids (Demirkan et al., PLoS Genetics, 2012) identified two novel loci supported by additional literature evidence: GPAM (Glycerol-3 phosphate acyltransferase) and CBS (Cystathionine beta-synthase). In addition, the workflow approach provided novel insight into the affected pathways and relevance of some of these gene-metabolite pairs in disease development and progression.

Conclusions: We demonstrate the utility of automated exploitation of background knowledge present in pathway databases for the analysis of GWAS datasets of metabolomic phenotypes. We report novel loci and potential biochemical mechanisms that contribute to our understanding of the genetic basis of metabolic variation and its relationship to disease development and progression.
\end{abstract}

Keywords: Genome-wide association, Metabolite, Genotype-phenotype prioritization, Bioinformatics,

Pathway databases

\footnotetext{
* Correspondence: P.A.C._t_Hoen@lumc.nl

${ }^{1}$ Center for Human and Clinical Genetics, Leiden University Medical Center, S4-P, PO Box 9600, 2300, RC Leiden, Netherlands

Full list of author information is available at the end of the article
}

\section{Biomed Central}

(c) 2013 Dharuri et al.; licensee BioMed Central Ltd. This is an open access article distributed under the terms of the Creative Commons Attribution License (http://creativecommons.org/licenses/by/2.0), which permits unrestricted use, distribution, and reproduction in any medium, provided the original work is properly cited. 


\section{Background}

GWAS have resulted in the identification of novel genetic loci associated with a variety of diseases and clinical phenotypes. However, a disease or clinical phenotype is the end point of the behaviour of numerous genes and pathways in addition to environmental influences. This at least partly explains the general observation that the effect size of genetic association with clinical phenotypes is rather small. Spurred by recent technological developments in the field of metabolomics, interest in genome wide association studies with metabolite levels in blood [1-4] is gathering momentum. Metabolites are intermediate phenotypes, entities that lie between genes and clinical end points $[5,6]$. Due to their proximity to an enzyme/gene, metabolites may offer greater effect sizes for GWAS than clinical phenotypes [7]. Moreover, the pathways in which the metabolite plays a role may provide insight into the underlying biological mechanism responsible for the development of the associated disease.

Typically, in metabolomics GWAS, hundreds of metabolites are tested for genetic association. However, association of all SNPs with all measured metabolites comes with considerable multiple testing problems. Recent publications have also shown that testing ratios of metabolites for genetic association results in much larger effect sizes; however this further exacerbates the multiple testing problem which precludes genuine SNPmetabolite pairs from reaching genome-wide significance. Several approaches like gene based tests $[8,9]$ and pathway analysis [10] have been proposed to overcome this limitation of inadequate statistical power in GWAS. All these approaches have been suggested in the context of GWAS with clinical phenotypes but genetic association with metabolites presents its own set of unique opportunities and challenges. Herewith, we explore the utility of background knowledge present in metabolic pathway databases to increase the power in identification of metabolite Quantitative Trait Loci (mQTL).

Our approach involves selective testing of SNPs near genes in pathways supposedly relevant to the metabolite levels, as a way to reduce the multiple testing burden in GWAS. Background knowledge pertaining to a metabolite is retrieved through systematic interrogation of metabolic pathway databases which describe biochemical pathways, reactions, and enzymes relevant to human metabolism. Several pathway databases have been created by groups around the world, while the intent of these efforts remains the elucidation of biological mechanism, the databases however, differ quite significantly in their content, size, user accessibility, download formats and most importantly availability and type of web services for machine-enabled interrogation of the database [11]. In this publication, as a proof of principle, we have chosen to focus on two important metabolic pathway databases, KEGG [12] and BioCyc [13]. KEGG is an integrated database resource of seventeen databases which provide system, genomic and chemical information. The pathway database consists of both metabolic and nonmetabolic pathways and is constructed by a team of curators based on information available in the literature. BioCyc is a collection of pathway/genome databases that describe the genome and metabolic pathways of several organisms. The database that describes human genomes and pathways, HumanCyc was interrogated in this study. In our approach, for every metabolite under consideration, genes acting in the vicinity of the metabolite are determined using knowledge present in databases mentioned above. We thus generate an integrated set of genes that represent entities with influence over the metabolite. A workflow management system called Taverna [14] was used to generate these gene sets and the SNPs associated with these genes. The workflows that were designed for this purpose have been submitted to a workflow repository at http://www.myexperiment.org/ packs/319.html [15].

A previously published metabolomics dataset by Illig et al. 2010 [2] was analyzed to evaluate the sensitivity of the method in picking true positives and to identify novel SNP-metabolite pairs that had hitherto been obscured in the GWA list given the stringent threshold for significance. In addition to validating a novel bioinformatics workflow analysis tool, we identified a new locus of human metabolic individuality, Aldehyde dehydrogenase family1 L1 (ALDH1L1). This locus was found associated with serine/glycine ratios, a metabolic trait that functionally matches the gene function.

Candidate genes identified through the analysis of Illig et al. dataset were taken up for replication in a separate study published by Demirkan et al. [4]. We report GPAM (Glycerol-3 phosphate acyltransferase) and CBS (Cystathionine beta-synthase) as novel loci associated with phosphatidylcholine moieties.

\section{Results}

Our approach can be divided into three stages: (i) Generate a non-redundant gene set for every metabolite considered using knowledge in pathway databases like KEGG and BioCyc applying interrogation schemes as shown in Figure 1 and outlined below. (ii) For every gene in the set, generate the set of SNPs within the gene and $50 \mathrm{~kb}$ flanking sequences, and create a SNP set for each metabolite (iii) Match SNPs generated for a metabolite with the GWAS for the same metabolite and store the matches with the p-values reported for the association (Figure 2).

\section{Analysis strategy of databases and Interrogation schemes} To retrieve a prioritized list of candidate genes associated with metabolite levels, gene sets were generated for 

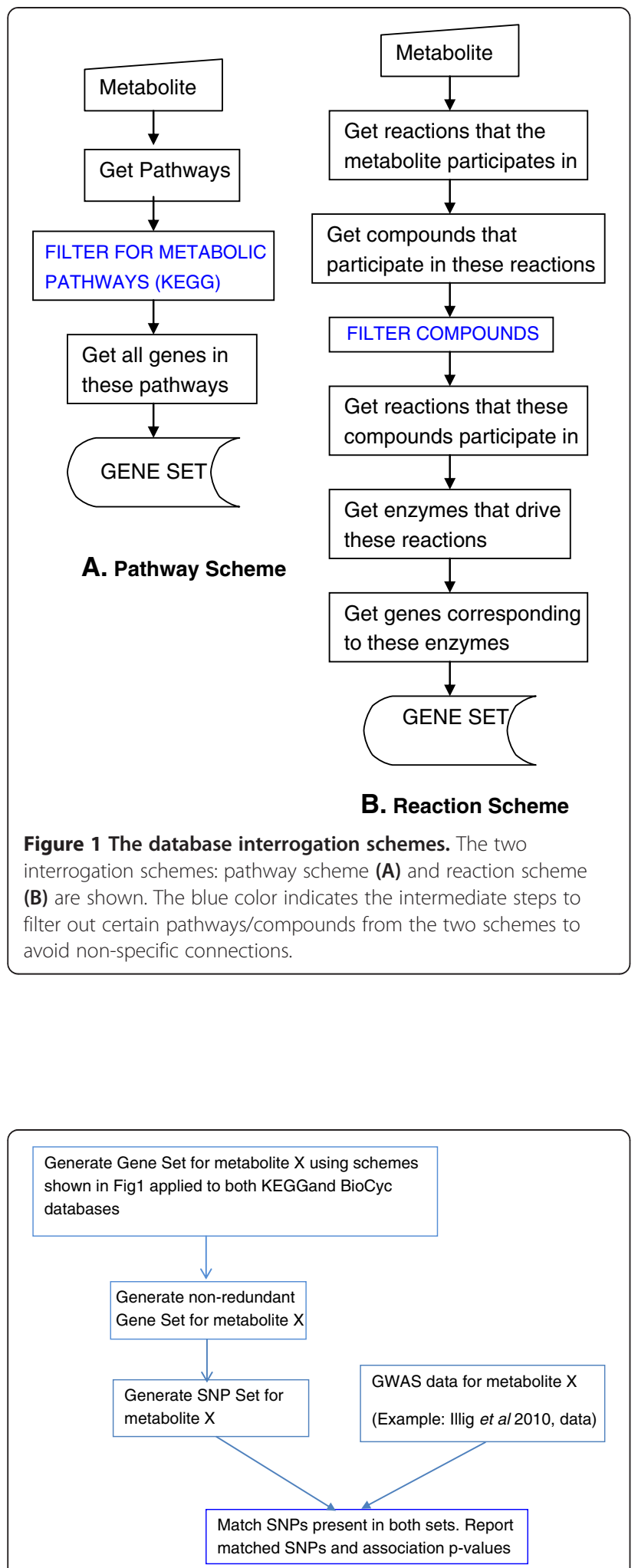

Figure 2 Strategy to find biologically relevant SNP-metabolite pairs in published GWAS datasets. Background knowledge pertaining to a metabolite is collected from the pathway databases KEGG and BioCyc in an automated fashion to generate a gene/SNP set relevant to the synthesis and degradation of the metabolite. each metabolite through the pathway scheme and the reaction scheme [Figure 1A and 1B] for the KEGG and BioCyc databases (see Method). The pathway scheme generates a list of genes that participate in pathways relevant to the synthesis or degradation of the metabolite. In the reaction scheme, the metabolite is used as a seed node and shells of reactions around the metabolite are explored. The list of genes that catalyse the reactions are retrieved and form the gene set for the given metabolite. For every gene set, a corresponding SNP set is generated by retrieving SNPs within the flanking $50 \mathrm{~kb}$ of every gene. In the final step, the SNP set for a metabolite is matched with the GWAS dataset for the same metabolite. At this stage, the sensitivity of the method is evaluated and potential novel discoveries are explored.

Results for each of three classes of metabolites (14 amino acids, 1 carnitine and 2 lipids) are shown in Table 1. For example, for glycine, interrogation of the KEGG database identified 173 and 432 genes using the pathway and reaction schemes respectively, whereas the corresponding numbers of genes were 90 and 192 for the BioCyc database. The union of all the four interrogation schemes results in a gene set consisting of 523 genes relevant to glycine metabolism (Table 1). For all the three classes of metabolites, 1246 unique genes were found, 640 are common to KEGG and BioCyc, the number of genes unique to each of the two databases are 379 and 227 respectively (Figure 3 ).

\section{Statistical threshold}

The number of unique SNPs generated for each of the metabolites is shown in Table 1. For aggregated metabolites like phosphatidylcholines, sphingomyelins and carnitines the size of the unique SNP set is multiplied by the number of metabolites that fall within each class to yield the total number of tests. For example, the size of the unique SNP set for carnitine is 11,239; this is multiplied by the number of carnitines which is 41 , to yield a total number of 460,799 tests for these compounds, as shown in the last column of Table 1 . The sum of all SNPs derived from our set of metabolites is 3,835,543. The multiple testing threshold for metabolite concentrations using a Bonferroni correction at a nominal p-value of 0.05 is $1.3 \mathrm{E}-08(0.05 / 3,835,543)$. In contrast, the pvalue threshold for significant association of SNPs with the same metabolite concentrations in the Illig et al. study would be $5.96 \mathrm{E}-10(0.05 / 162 * 517,840)$. This represents a reduction of the multiple testing burden by about two orders of magnitude, regardless of the dependency between the SNPs or metabolites.

It has been demonstrated that GWAS of metabolite ratios offer robust statistical associations and point to biological mechanisms related to the interconversion of metabolite pairs. To investigate the association of SNPs 
Table 1 Gene and SNP sets generated by the database: interrogation schemes for each of the metabolites

\begin{tabular}{|c|c|c|c|c|c|c|c|}
\hline Metabolite & $\begin{array}{l}\text { BioCyc } \\
\text { Pathway }\end{array}$ & $\begin{array}{l}\text { BioCyc } \\
\text { Reaction }\end{array}$ & $\begin{array}{l}\text { KEGG } \\
\text { Pathway }\end{array}$ & $\begin{array}{l}\text { KEGG } \\
\text { Reaction }\end{array}$ & $\begin{array}{l}\text { Size of unique } \\
\text { gene set }^{1}\end{array}$ & $\begin{array}{l}\text { Size of unique } \\
\text { SNP } \text { set }^{2}\end{array}$ & $\begin{array}{l}\text { Number } \\
\text { of tests }^{3}\end{array}$ \\
\hline Arginine & 20 & 104 & 57 & 179 & 257 & 10788 & 10788 \\
\hline Glutamine & 51 & 132 & 100 & 282 & 388 & 15591 & 15591 \\
\hline Glycine & 90 & 192 & 173 & 432 & 523 & 20767 & 20767 \\
\hline Histidine & 8 & 9 & 45 & 155 & 181 & 7126 & 7126 \\
\hline Leucine & 8 & 0 & 44 & 83 & 117 & 5037 & 5037 \\
\hline Methionine & 27 & 104 & 35 & 243 & 284 & 11532 & 11532 \\
\hline Ornithine & 16 & 150 & 103 & 159 & 247 & 10089 & 10089 \\
\hline Phenylalanine & 6 & 113 & 25 & 163 & 196 & 8419 & 8419 \\
\hline Proline & 10 & 12 & 57 & 83 & 119 & 5075 & 5075 \\
\hline Serine & 37 & 135 & 152 & 219 & 360 & 14996 & 14996 \\
\hline Threonine & 1 & 11 & 39 & 49 & 75 & 2633 & 2633 \\
\hline Tryptophan & 15 & 19 & 78 & 221 & 261 & 10419 & 10419 \\
\hline Tyrosine & 14 & 106 & 61 & 158 & 219 & 9365 & 9365 \\
\hline Valine & 15 & 93 & 80 & 137 & 211 & 9365 & 9365 \\
\hline Carnitine & 32 & 206 & 81 & 94 & 263 & 11239 & 460799 \\
\hline Phosphatidylcholine & 188 & 361 & 312 & 343 & 640 & 31676 & 2914192 \\
\hline Sphingomyelin & 160 & 331 & 189 & 241 & 460 & 21290 & 319350 \\
\hline Sum & 698 & 2078 & 1631 & 3241 & 4801 & 205407 & 3835543 \\
\hline Unique set & 399 & 806 & 703 & 768 & 1246 & 55952 & 55952 \\
\hline
\end{tabular}

The number of genes for each metabolite and the corresponding database:interrogation scheme is shown. ${ }^{1}$ The size of the union of the gene set obtained from all the four database:interrogation schemes. ${ }^{2}$ The size of the corresponding SNP set. ${ }^{3}$ The number of tests is the same as the size of the SNP set for the amino acids whereas for aggregated entities like the lipids and carnitine the SNP set is multiplied by the number of compounds present in that class.

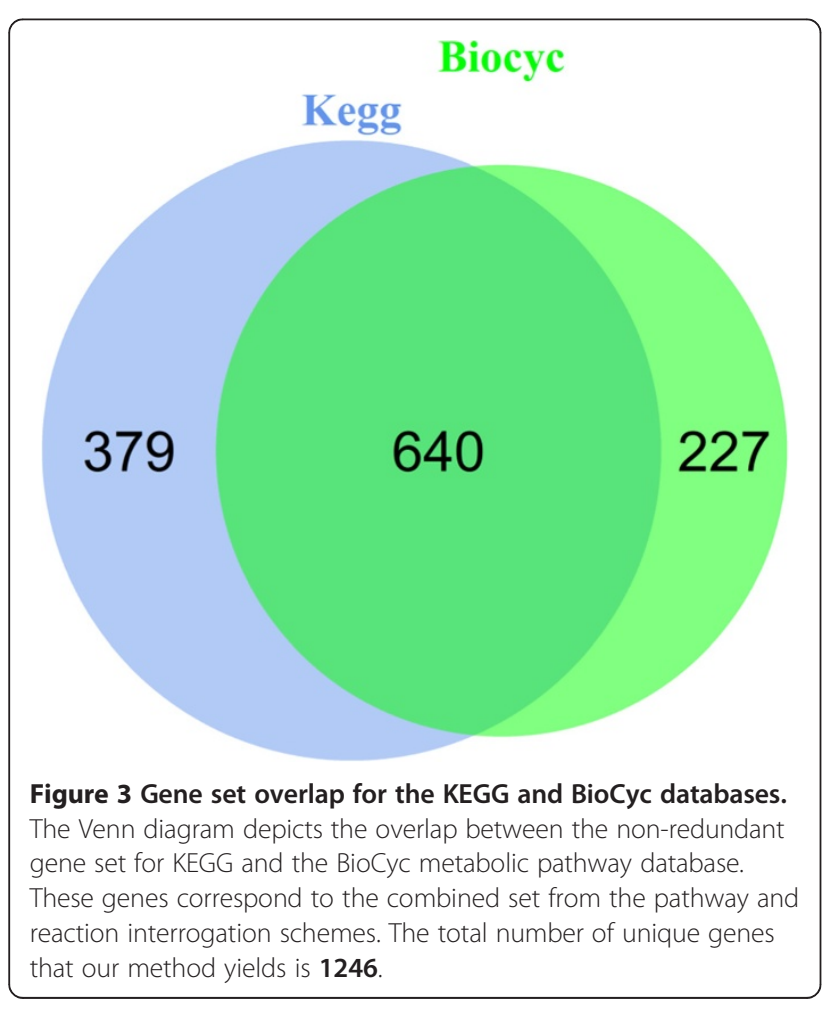

with metabolite ratios, we generated the union of SNP sets for all combinations of metabolites (Additional file 1: Table S3). In the case of aggregated metabolites like the lipids and carnitines, the union of the SNP set is multiplied by the number of compounds that fall within each class. For example, the union of the SNP set for arginine and carnitine is 20,000 , this is multiplied by 41 to yield the total number of 820,000 tests for this group of ratios. The number of tests for ratios of compounds within classes such as phosphatidylcholines is equal to the size of the unique SNP set multiplied by the number of combinations, $n^{*}(n-1) / 2$, which in this case would be $92 * 91 / 2=4186$. In choosing combinations of ratios, we have assumed that the association $\mathrm{p}$-value for a linear regression model using a metabolite ratio of $\mathrm{A} / \mathrm{B}$ is equivalent to that computed using it's reciprocal, B/A. The evidence for lack of independence of a ratio and its reciprocal is provided by the Illig et al. study where a comparison of associations computed using untransformed and log-scaled ratios did not detect significant differences. This implies that we may consider the p-values computed using $\mathrm{A} / \mathrm{B}$ and $\mathrm{B} / \mathrm{A}$ to be approximately equal.

The sum of the number of tests for all ratios is 423,645,558 as shown in Additional file 1: Table S3. The multiple testing threshold for the ratios using Bonferroni correction at nominal p-value of 0.05 is $1.18 \mathrm{E}-10$. This 
represents a multiple threshold reduction by two orders of magnitude over the genome-wide threshold estimated by Illig et al. which is $3.63 \mathrm{E}-12$.

\section{Proof of principle: sensitivity}

The sensitivity of the method was evaluated based on its ability to identify the top hits in the previously published Illig et al. genome-wide association study. The overall sensitivity of the method as well as the interrogation specific breakdown is shown in Table 2. For example, for the BioCyc pathway scheme the size of the unique gene set generated for all the metabolites is shown to be 399. The number of genes that are among the 15 top hits in the Illig et al. study for this database:interrogation scheme is 8 which results in a sensitivity measure of 0.53 . A metabolite specific breakdown of each of these schemes and the genes with a p-value cutoff of 1E-02 is shown in Additional file 1: Table S5.

Overall, combining the results from the four database: interrogation schemes helped identify 10 of the 15 top associations (67\% sensitivity) published by Illig et al.

\section{Novel discovery in the Illig et al dataset}

Analysis of the first stage or the "discovery stage" dataset of 1029 samples from the Illig et al. dataset yielded several associations with $\mathrm{p}$-values indicative for association, but that did not meet the significance threshold applied by Illig et al. Associations with p-value less than 1E-02 were evaluated in the combined "replication stage" dataset with 1809 samples. Analysis of SNPs in the ALDH1L1 (aldehyde dehydrogenase family $1 \mathrm{~L} 1$ ) gene locus lowered the $\mathrm{p}$-value of association with serine/glycine ratio from 4.83E-09 in the discovery dataset to 5.13E-12 in the combined dataset. This is well below our threshold of 1.18E10 , but above the threshold to be applied when considering all associations between SNPs and metabolite ratios. Furthermore, the original publication did not select this association for replication because of the threshold set in the first stage of the analysis. This is an example of the method pointing to potential true positives in a genomewide scan and the association of ALDH1L1 with the trait is being reported as a novel discovery.

\section{Statistical threshold in the replication study}

The analysis of the Illig et al. dataset identified several biologically relevant candidate genes with $\mathrm{p}$-values less than 1E-02. A list of 56 of these genes associated with phosphatidylcholines and sphingomyelins were investigated in an independent study in the GWAS dataset of phospholipids published by Demirkan et al. The number of matches between the two datasets was: 56 phosphatidylcholines and 6 sphingomyelins. Demirkan et al. also performed GWAS for within class molar proportions for these moieties. We took these into consideration in addition to the GWAS of absolute concentrations. Therefore, the total number of metabolites and proportions investigated in the Demirkan et al. GWAS dataset was 124. A principal component analysis based on the method proposed by $\mathrm{Li}$ et al. [16] was performed on this set of metabolites resulting in 51 effectively independent variables. As we considered 2413 independent SNPs in the candidate loci for these metabolites, the statistical threshold, applying Bonferroni correction at a nominal p-value of 0.05 , for the replication study was 4.06E-07 (0.05/2413*51).

\section{Novel discoveries in the replication study}

Table 3 shows the top hits in the meta-analysis of candidate genes identified in the Illig et al. dataset for replication. The meta-analysis was performed using Stouffer's Zscore based method of combining p-values [17]. Since the SNPs in the loci replicated in the Demirkan et al. dataset had relatively low $\mathrm{r}^{2}$ values with the SNPs reported in the Illig et al. dataset, we could not perform a traditional meta-analysis where strict linkage disequilibrium criteria are applied. Therefore, we combined the lowest p-value per gene and sought additional supporting evidence for potential allelic heterogeneity (see Discussion). As mentioned earlier, the p-value threshold for the replication study is set at 4.06E-07. SNPs in the vicinity of the genes CBS, GPAM, ADCY8, CNR1, HSD17B12, MBOAT1, PECR, PLCB1 and TECR pass this threshold.

\section{Discussion}

Genome wide association studies with metabolites as phenotypes have identified several loci that explain

Table 2 Performance of the database:interrogation schemes in GWAS dataset analysis

\begin{tabular}{|c|c|c|c|}
\hline Database: interrogation scheme & Size of gene set ${ }^{1}$ & Top hits from Illig et al. study identified by the method ${ }^{2}$ & Sensitivity $^{3}$ \\
\hline BioCyc pathway & 399 & ACADL, ACADM, ACSL1, CPS1, FADS1, PHGDH, SCD, SPTLC3 & 0.53 \\
\hline BioCyc reaction & 806 & ACADM, ACADS, ACSL1, CPS1, FADS1, SCD, SPTLC3 & 0.47 \\
\hline KEGG pathway & 703 & ACADL, ACADM, ACADS, ACSL1, CPS1, ELOVL2, FADS1, PHGDH, SCD, SPTLC3 & 0.67 \\
\hline KEGG reaction & 768 & ACADL, ACADM, ACADS, ACSL1, CPS1, PHGDH, SCD, SPTLC3 & 0.53 \\
\hline Pooled set & 1246 & ACADL, ACADM, ACADS, ACSL1, CPS1, ELOVL2, FADS1, PHGDH, SCD, SPTLC3 & 0.67 \\
\hline
\end{tabular}

Snapshot of the matches between our method and the association data from the Illig et al. 2010 study for each of the database:interrogation scheme. ${ }^{1}$ corresponds to the unique set of genes generated for all the metabolites for the given database:interrogation scheme. ${ }^{2}$ corresponds to the top hits in the Illig et al. publication that were present in the gene set for the given database:interrogation scheme. ${ }^{3}$ Sensitivity is a measure of the actual positives that have been captured by our method and is equal to the ratio of the number of top hits identified by the method over the total number of top hits in the Illig et al. publication which is 15 . 
Table 3 Replication of candidate genes in the Demirkan et al. dataset

\begin{tabular}{|c|c|c|c|c|c|c|}
\hline Gene & Trait & $\begin{array}{l}\text { SNP from the } \\
\text { Illig et al. } \\
\text { dataset }\end{array}$ & p-value ${ }^{1}$ & $\begin{array}{l}\text { SNP from the } \\
\text { Demirkan } \\
\text { et al. dataset }\end{array}$ & p-value ${ }^{2}$ & $\begin{array}{l}\text { Combined } \\
\text { p-value }\end{array}$ \\
\hline ADCY8 & PC ae $C 40: 6$ & rs11786743 & 4.03E-05 & rs913819 & $6.73 \mathrm{E}-04$ & $2.15 \mathrm{E}-07$ \\
\hline$C B S^{*}$ & PC ae $C 40: 6$ & rs2839631 & 5.67E-06 & rs378376 & $5.17 \mathrm{E}-04$ & $2.90 \mathrm{E}-08$ \\
\hline CNR1 & PC ae C38:2 & rs10485168 & $2.42 \mathrm{E}-04$ & rs9359765 & 4.61E-04 & $7.54 \mathrm{E}-07$ \\
\hline GPAM ${ }^{*}$ & PC ae C34:3 & rs2246253 & $1.25 \mathrm{E}-04$ & rs2419603 & $1.76 \mathrm{E}-04$ & $1.56 \mathrm{E}-07$ \\
\hline HSD17B12 & PC aa C34:4 & rs2862999 & $2.66 \mathrm{E}-05$ & rs11037685 & $6.13 \mathrm{E}-04$ & $1.35 \mathrm{E}-07$ \\
\hline MBOAT1 & PC ae $C 40: 6$ & rs9465673 & $1.11 \mathrm{E}-04$ & rs694094 & 4.47E-04 & $3.53 \mathrm{E}-07$ \\
\hline PECR & PC aa C38:0 & rs3770536 & $5.55 \mathrm{E}-04$ & rs3770562 & $9.43 \mathrm{E}-05$ & 3.79E-07 \\
\hline PLCB1 & PC aa C30:0 & rs6056188 & $9.55 \mathrm{E}-06$ & rs17363114 & $1.96 \mathrm{E}-03$ & $2.06 \mathrm{E}-07$ \\
\hline TECR & PC aa C32:0 & rs7252966 & $1.69 \mathrm{E}-05$ & rs7254215 & 2.09E-03 & $3.57 \mathrm{E}-07$ \\
\hline
\end{tabular}

Top hits from the meta-analysis of candidate genes identified in the Illig et al. study and replicated in the Demirkan et al. dataset. ${ }^{1,2,3}$-value of association of the SNP with the trait in the Illig et al., Demirkan et al. and combined p-value respectively. *indicates genes for which further evidence was found.

human metabolic individuality. However, the large metabolite panel being tested results in a severe multiple testing burden that precludes genuine SNP-metabolite pairs from consideration when they fail to reach the stringent threshold for statistical significance. Our method aims to address this problem by selectively testing genes that operate in reactions and pathways relevant to the metabolite. The goal is to reduce the severity of the multiple testing burden and identify potential true positives in the list of genome-wide associations. Taverna, a workflow management system was used to generate the SNP-metabolite pairs. We have deposited the workflows at a repository called myexperiment.org, making it easier for the scientific community to interpret, repeat and reproduce the result. The sensitivity of the method, defined as retrieval of previously identified associations, is high, as evident from the proof of principle study carried out on the genome scan published by Illig et al. Replication studies on some of the promising SNPmetabolite pairs identified by the method pointed to a novel and statistically significant association at the ALDH1L1 locus with serine/glycine ratios. Additional replication studies of phosphatidylcholines and sphingomyelins uncovered significant gene-wise associations with CBS, GPAM, ADCY8, CNR1, HSD17B12, MBOAT1, PECR, PLCB1 and TECR.

\section{Databases, interrogation schemes and software tool}

The pathway databases have technical and conceptual differences [11] that mandate interrogation of multiple databases and integration of the results. Interpretation of these results requires a close coordination between biologists and computer scientists. Workflow management systems in general and Taverna [Additional file 1: S2] in particular is an example of a software tool that is intuitive enough for the biologist, while at the same time offering the flexibility for exploring the algorithmic aspects for the computer scientist [18]. In using Taverna as a software tool and depositing the workflows in the repository myexperiment.org, we have attempted to make the method and the rationale transparent to users, thus facilitating its retrieval, reuse and reproduction by other independent scientists [19].

\section{Sensitivity of the method}

As a sensitivity measure of our method, we evaluated its ability to pick the top hits in the Illig et al. publication [2]. Some $60 \%$ of the top associations were identified successfully. A similar analysis of GWAS dataset published by Suhre et al. [3] yielded a sensitivity of 54\% (20 out of 37 hits) (data not shown). However, 4 of the "misses" in the Suhre et al. dataset were peptide fragments that do not have an entry in the pathway databases, which is a prerequisite for our method to work.

We interpret the high sensitivity of our method in three ways; first it reinforces the rationale that GWAS with metabolomic phenotypes provides a functional approach to the study of human genetic variation [1]. In other words, the known function of the associated gene and the biochemical characteristics of the affected metabolite support each other in ways that lends itself to a narrative on the underlying biological mechanism. Second, while the pathway databases have a long way to go in achieving a comprehensive annotation and delineation of biological processes, they, however, are a good resource of information in so far as the top hits in a GWAS with metabolomic phenotypes are concerned. Only two out of the 15 top hits in the study by Illig et al. were genes with unknown functions (PLEKHH1,SYNE2), and two others were hitherto uncharacterized solute transporters (SLC16A9, SLC22A4). Third, a good sensitivity measure is a validation of our method and reflects its comprehensive data collection ability through integration of disparate data sources and utilization of appropriate interrogation strategies. 


\section{Novel discoveries}

Our analysis of the GWAS dataset of the Illig et al. publication based on the first step of the "discovery design" yielded several interesting associations that had not been reported among the top hits in the publication. We selected a few of the promising associations for replication in the combined dataset of 1809 subjects. One of the genes, Aldehyde dehydrogenase family 1 L1 (ALDH1L1) was found associated with the ratio of serine/glycine with a p-value of 5.13E-12 in the combined set of 1809 subjects. ALDH1L1 also known as 10-formyltetrahydrofolate dehydrogenase (10-FTHFDH, FDH) catalyzes the $\mathrm{NADP}^{+}$ dependent oxidation of 10-formyltetrahydrofolate to $\mathrm{CO}_{2}$ and tetrahydrofolate (THF) [20] as shown in Figure 4. It plays an important role in folate metabolism [21-25]. Among other functions, $A L D H 1 L 1$ has been known to deplete cellular 10-formyltetrahydrofolate pool resulting in a loss of de novo purine biosynthesis [23], maintain cellular folate concentrations by regulating the availability of THF [22], but most importantly, it has been shown to compete with the enzyme serine hydroxymethyl transferase (SHMT) for the polyglutamyltetrahydrofolates [25]. The latter enzyme catalyzes the conversion of serine to glycine as shown in Figure 4. It has also been shown that glycine to serine inter-conversion by SHMT accounts for approximately $41 \%$ of whole body glycine flux inclusive of both mitochondrial and cytoplasmic processes [26].

To further investigate the potential of our approach to uncover novel genetic associations, we extended the analysis to an additional independent GWAS dataset [4]. Candidate genes identified in the Illig et al. dataset in association with phosphatidylcholines and sphingomyelins were considered for replication in the dataset provided by Demirkan et al. [4]. We discuss here two novel findings for which additional evidence was obtained.
SNPs near glycerol-3 phosphate acyltransferase (GPAM) are associated with PC ae C34:3 moieties in the Illig et al. and Demirkan et al. datasets with p-values of $1.25 \mathrm{E}-04$ and $1.75 \mathrm{E}-04$, respectively, with a meta-analysis p-value of 1.56E-07. GPAM encodes a mitochondrial protein that esterifies the acyl group from acyl-coA to the sn-1 position of glycerol-3-phosphate. It is a ratelimiting enzyme that catalyzes the initial step in the biosynthesis of triacylglycerols and phospholipids [27]. A recent study showed that in breast cancer, GPAM expression is strongly correlated with survival rates, clinico-pathological features as well as metabolomic and lipidomic profiles [28]. Interestingly, the study identified the metabolite PC C34:3 as the most significantly altered metabolite with respect to GPAM expression in breast cancer patients. This suggests that, for this particular example, genetic control is primarily at the level of gene expression, with secondary effects on enzyme levels and metabolic conversion rates. The example also highlights the potential influence of genetic variation of metabolic pathways on disease.

A large number of genes identified by our method in the context of phospholipids participate in fatty acid metabolism and are therefore likely to affect the levels of groups of phosphatidylcholines and sphingomyelins. For example, GPAM esterifies the acyl group from acyl-ACP to the sn-1 position of glycerol-3-phosphate, and is therefore relevant to both acyl-acyl and acyl-alkyl moieties. The lowest p-value of association, at this locus, with a phosphatidylcholine moiety in the Illig et al. study is with PC ae C36:3, while in the Demirkan et al. study it is $\mathrm{PC}$ aa C36:3. Since both associations make biological sense, future work should incorporate joint modelling of suitable phospholipid moieties to help identify loci that are biologically relevant but fail to reach the statistical

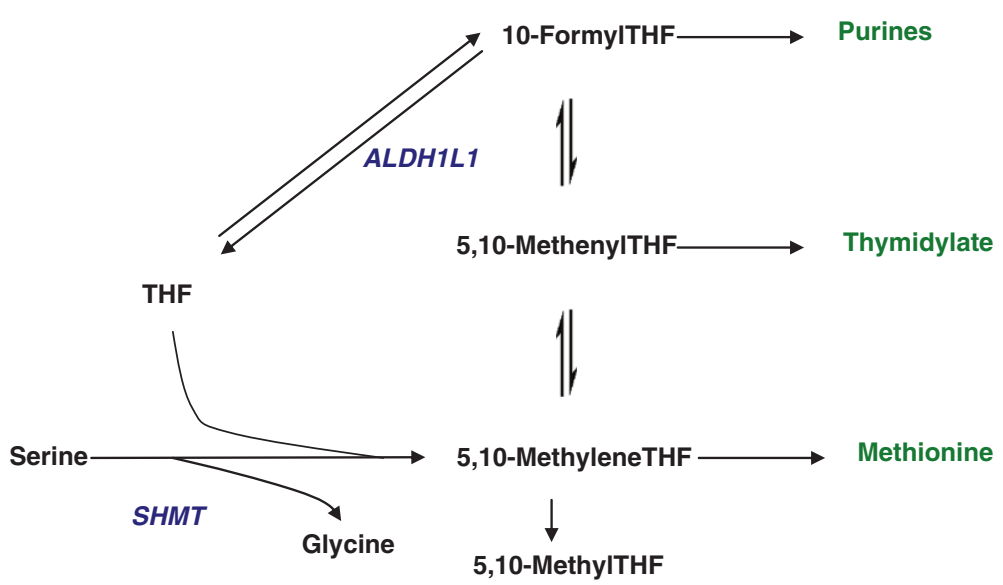

Figure 4 Role of ALDH1L1 in the cytosolic one-carbon pool metabolism. A simplified schematic of the one-carbon pool metabolism in the cytosol is depicted. ALDH1L1: Aldehyde Dehydrogenase 1 Family, Member L1; THF: tetrahydrofolate; SHMT: Serine hydrxymethyltransferase. 
threshold in GWAS analysis. We have reported such best case associations for phosphatidylcholines in Additional file 1: Table S6.

SNPs near Cystathionine beta-synthase (CBS) are associated with PC ae C40:6 moieties in the Illig et al. and Demirkan et al. datasets with p-values of 5.67E-06 and 5.17E-04, respectively, with a meta-analysis p-value of 2.9E-08. Mutations in CBS cause hyperhomocysteinemia [29], which is marked by elevated levels of homocysteine. Several studies have associated altered phosphatidylcholine biosynthesis with hyperhomocysteinemia/ CBS deficiency [30-33]. In one of the studies [30], phosphatidylcholine levels and the activity of the enzyme lecithin-cholesterol acyltransferase $(L C A T)$ were significantly lower in $C B S$ deficient mice than in wild type mice. While there is considerable literature evidence for the role of $C B S$ in phosphatidylcholine metabolism, the stringent p-value threshold obscures this association in the list of GWAS results.

The low $\mathrm{r}^{2}$ values for significant SNPs in GPAM, CBS and other loci between the Illig et al. and Demirkan et al. datasets could be explained by allelic heterogeneity. The latter is a phenomenon where multiple alleles from one gene influence a trait. However, in some cases it may be that the two apparently independent SNPs are tagging a third SNP [34]. This may be the case for the two SNPs (rs2839631, rs378376) near CBS which have an $r^{2}$ of 0.067 and are associated with C40:6 phosphatidylcholines in both the datasets. However, both SNPs are in LD with cis-eQTLs in the region (for example, rs719037, $\left.\mathrm{r}^{2} \sim 0.4\right)$. This is suggestive of the SNPs exerting their effect through the expression levels of the $C B S$ enzyme, as was suggested for GPAM. Apparent allelic heterogeneity may preclude identification in a standard meta-analysis, but would justify further investigation of independent or dependent signals at loci showing this phenomenon.

\section{Challenges and future direction}

In general, our effort was directed at exploring the utility of machine-enabled interrogation of metabolic pathway databases in prioritizing SNP-metabolite associations in a GWAS dataset. While the method's sensitivity and ability to make novel discovery are encouraging, considerable progress needs to be made in metabolite disambiguation to achieve a relevant and comprehensive gene set for a given metabolite. This problem is particularly acute for phospholipids like phosphatidylcholines and sphingomyelins and various forms of the fatty acid transporters of L-carnitine. For example, the metabolomics technology used in the Illig et al. study differentiated more than 90 forms of phosphatidylcholines based on alkyl or acyl bonds and single or double bonds on the side chains. However, the pathway databases do not yet contain information for the complex structures. This forces users to analyze these metabolites at a higher aggregation level. Another issue that requires attention is the bias introduced in selecting genes for inclusion in the gene set. We have formulated simple rules for interrogation [Additional file 1: S1] that facilitates unbiased generation of gene sets for any given metabolite.

Another challenge arises due to the high correlation between metabolites, particularly the phospholipids like phosphatidylcholines and sphingomyelins. These moieties are associated with loci relevant to fatty acid metabolism. While the variation at these loci effects the levels of fatty acids and thereby the phospholipid pool, to a large extent, these loci are not specific for any particular phospholipid moiety. As a result, several loci exhibit a pleiotropic effect for biologically related metabolic phenotypes in general and phospholipids in particular [Shown in Additional file 1: Table S7] We have demonstrated that background knowledge and evidence-based approach is ideally suited to identify such candidate genes, however future work should focus on statistical methodologies with sufficient power to detect such pleiotropic loci in GWAS of intermediate phenotypes. In summary, future work includes integration of more pathway databases, metabolite disambiguation, consideration of allelic heterogeneity and multivariate statistical techniques that take into account the high degree of correlation between the metabolites.

\section{Conclusions}

A measurement of metabolites as intermediate phenotypes is a potentially powerful approach to uncover the influence of genetic variation on disease susceptibility and progression. However, we still face many hurdles in the interpretation of GWAS data. In this study, we investigated the utility of background knowledge present in pathway databases in extending our understanding of the genetic basis of metabolic variation. We developed a bioinformatics method that prioritizes SNP-metabolite associations in a GWAS based on metabolic pathway information present in the KEGG and BioCyc databases. The validity of the method is demonstrated by reanalysing published GWAS datasets and identifying previously known associations. We report a new locus of human metabolic individuality, ALDH1L1 (Aldehyde dehydrogenase family $1 \mathrm{~L} 1$ ) associated with serine/glycine ratios. Replication studies in an independent GWAS of phospholipids identified GPAM (Glycerol-3 phosphate acyltransferase) and CBS (Cystathionine beta-synthase) as novel loci, and this was further supported by additional literature evidence. The utility of a workflow management system in facilitating novel biological discoveries and as a tool for efficient sharing of computational protocols is demonstrated. 


\section{Methods}

\section{GWAS data set for proof of principle studies}

The GWAS dataset published by Illig et al. 2010 [2] was used to evaluate the validity of the method. Illig et al. employed a two-stage discovery design in the KORA F4 population cohort with 1029 male and female individuals in the first stage and 780 individuals in the second stage. Loci with $\mathrm{p}$-value of association $<10^{-7}$ for metabolite concentrations and $\mathrm{p}$-value $<10^{-9}$ for concentration ratios were taken up for the second stage independent testing in 780 individuals. The joint p-values of association for all the 1809 individuals were then computed and 15 loci were reported whose strength of association increased after the second stage of the discovery process. The authors note that "although this approach is less well powered than a full genome-wide joint analysis, it reflects the historical way in which [they] selected SNPs for follow-up". This means that if we can identify potential true positives using the 1029 samples, we can validate them in the full dataset, since this has not been done in the Illig et al. study for all hits with p-value $>10^{-7}$ for metabolite concentrations and $\mathrm{p}$-value $>10^{-9}$ for concentration ratios. Therefore, the GWAS dataset based on 1029 samples was analyzed for our proof of principle studies. Additionally, to evaluate novel associations identified by the method in the discovery stage dataset, the strength of the signal was assessed in the combined GWAS dataset for 1809 subjects.

\section{GWAS dataset for follow-up studies}

Candidate loci identified in the Illig et al. dataset by our method were taken up for follow-up studies in the dataset published by Demirkan et al. The latter conducted a metaanalysis of GWAS on plasma levels of ceramides, phosphatidylcholines, lysophosphatidylcholines, sphingomyelins, phosphatidylethanolamines and plasmalogens in five European populations: the Erasmus Rucphen Family (ERF) study, conducted in the Netherlands, (2) the MICROS study from the Tyrol region in Italy, (3) the Northern Swedish Population Health Survey (NSPHS) in

Norrbotten, Sweden, (4) the Orkney Complex Disease Study (ORCADES) in Scotland, and (5) the CROAS (CROATIA_Vis) study conducted on Vis Island, Croatia. Broadly, the metabolite overlap between the Illig et al. dataset and Demirkan A et al. dataset was confined to the class of phosphatidylcholines, lysophosphatidylcholines and sphingomyelins. More specifically, the overlap represented 62 phospholipid moieties. Also, 56 candidate genes were identified for follow up in the Illig et al. dataset. We choose to focus on SNPs in the flanking $50 \mathrm{~kb}$ region of these genes for the follow-up study in the Demirkan A et al. dataset.

\section{Metabolites considered for the generation of gene sets}

Gene sets are defined as entities that participate in pathways and reactions relevant to the metabolite and hence hold the potential to influence its levels. The goal was to generate gene sets for the compounds that were measured in the Illig et al. 2010 publication: 14 amino acids (Arginine, Glutamine, Glycine, Histidine, Methionine, Ornithine, Phenylalanine, Proline, Serine, Threonine, Tryptophan, Tyrosine, Valine, and Leucine), 41 Carnitines, 92 Phosphatidylcholines and 15 Sphingomyelins. In addition to the metabolites mentioned above, Illig et al. also measured Hexose. We did not consider this metabolite for investigation because pathway information surrounding hexose is lacking. While metabolites like glucose and fructose could have been considered as proxies, we did not pursue this because of the enormous size of the resulting gene set, combined with a lack of confidence in the relevance of many of these genes to the metabolite measured by the metabolomics platform.

\section{Pathway databases and interrogation schemes}

The metabolic pathway databases KEGG (release 63) [12] and BioCyc (version 16) [13] were accessed for retrieving background knowledge surrounding metabolites. Two interrogation schemes were employed: pathway scheme and reaction scheme (Figure 1). In a pathway scheme, for a given metabolite, all the pathways that it participates in are determined followed by the retrieval of all the genes that participate in these pathways (Figure 1A). In a reaction scheme, given a metabolite, all the reactions that it is part of and the compounds that participate in these reactions are determined. The compounds obtained at this point are subjected to the same strategy as in the previous step in that all the reactions that these compounds participate in are determined. This can be visualized as expanding by a radius of 2 steps in the reaction space of every metabolite. Finally, the enzymes that drive all these reactions are determined (Figure 1B). As an intermediate step certain compounds were filtered out in order to avoid non-specific connections. The details about the filtration step and the compounds that were filtered are provided in the Additional file 1: S4. In all there are four schemes: kegg:pathway, kegg:reaction, biocyc:pathway, and biocyc:reaction. The set of non-redundant genes combined from all the schemes then forms the gene set for any given metabolite.

\section{Software used to generate gene and SNP sets}

Taverna version 2.4 [14], a workflow management system was used to generate metabolite specific gene sets as well as for the generation of SNPs present in the $50 \mathrm{~kb}$ flanking region of each gene. Taverna allows users access to remote data resources like KEGG, BioCyc, Ensembl, NCBI etc. and data management systems like Biomart through implementation of web services. Each component in a workflow is responsible for a particular function and many such components need to be chained 
together in a pipeline to create a workflow that performs a certain task. The pipeline depicted in Figure 1 is implemented in a Taverna workflow through appropriate linking of remote web services and local scripts. Web services are software systems that facilitate machine to machine interaction over a network. Taverna allows the inclusion of different kinds of web services like Web Services Description Language (WSDL) and REpresentational State Transfer (REST). The services provided by the KEGG database were implemented using the REST services made available in the Taverna workbench. The BioCyc database was accessed through the REST interface using the BioVelo language. The latter is a query language designed to let the users write precise queries against the pathway/genome databases, available at BioCyc, to retrieve pathways, reactions, compounds, genes etc. All the workflows were designed following best practices for workflow design [35].

\section{Workflow accessibility}

To facilitate retrieval and reproducibility, the workflows have been deposited in a repository at http://www.myexperiment.org/packs/319.html. While the focus of this paper was on a specific set of metabolites; using appropriate identifiers from the KEGG or BioCyc database users will be able to generate gene sets for other metabolites. To generate a gene set for any metabolite using the KEGG or BioCyc database, users have to input the metabolite identifier for that database and the output is a text file containing the entrez gene identifiers. For example, to generate a gene set for the metabolite Arginine, for either the pathway or reaction scheme using the KEGG database, users input the KEGG identifier for Arginine: C00062. Similarly, to obtain a gene set using the BioCyc database, the input for the same metabolite is "L-arginine". The workflows may also be repurposed to suit other objectives, for example, to filter out nonspecific connections, we remove hub metabolites like ATP, NADP and other entities like co-enzymes; however, users may change the filtration criteria if they find it too stringent for their objectives. A detailed tutorial on how to access and run these workflows is provided in the Additional file 1: S2.

\section{Additional file}

Additional file 1: S1. Rules to generate Metabolite-Gene sets. S2. Taverna workflow management system. Figure S1. Snapshot of the Taverna workbench which consists of three panels as pointed to in the figure. Table S3. SNP set generated for ratios of metabolites. S4. Compounds filtered for the Kegg:Reaction Scheme. Table S5. Metabolite specific break-up of the performance of database:interrogation schemes. Table S6. Best case associations of loci with phosphatidylcholines in the Illig et al and Demirkan et al datasets. Table S7. Pleiotropic effect for phosphatidylcholines at select loci.

\section{Competing interests}

The authors declare that they have no competing interests.

\section{Authors' contributions}

$\mathrm{HD}$ created the workflows and the algorithms to analyze GWAS datasets. HD, $\mathrm{PH}, \mathrm{KWvD}$, and PACtH prepared the manuscript. PH, KWvD and PACtH contributed ideas and supervised the project. JK contributed towards the analysis of GWAS datasets. MR, KH assisted in design of workflows. DMK, CG, RWG, JA, KS, AD, CMD provided the data sets for this study. All authors read and approved the final manuscript.

\section{Acknowledgments}

This study is funded by the European Community's Seventh Framework Programme (FP7/2007-2013) ENGAGE, the Centre for Medical Systems Biology (CMSB) and Netherlands Consortium for Systems Biology (NCSB), both within the framework of the Netherlands Genomics Initiative (NGI)/ Netherlands Organisation for Scientific Research (NWO) and the European Commission Seventh Framework Programme Wf4Ever (Digital Libraries and Digital Preservation area ICT-2009.4.1 project reference 270192). EUROSPAN consortium members are: Ayşe Demirkan, Cornelia M van Duijn, Peter Ugocsai, Aaron Isaacs, Peter P Pramstaller,Gerhard Liebisch, James F Wilson, Åsa Johansson, Igor Rudan, Yurii S Aulchenko, Anatoly V Kirichenko, A Cecile JW Janssens, Ritsert C Jansen, Carsten Gnewuch, Francisco S Domingues, Cristian Pattaro, Sarah H Wild, Inger Jonasson, Ozren Polasek, Irina V Zorkoltseva, Albert Hofman, Lennart Karssen, Maksim Struchalin, James Floyd, Wilmar Igl, Zrinka Biloglav, Linda Broer, Arne Pfeufer, Irene Pichler,Susan Campbell, Ghazal Zaboli, Ivana Kolcic, Fernando Rivadeneira, Jennifer Huffman, Nicholas D Hastie, Andre Uitterlinden, Lude Franke, Christopher S Franklin, Veronique Vitart, Jacqueline CM Witteman, Tatiana Axenovich, Ben A Oostra, Thomas Meitinger, Andrew A Hicks, Caroline Hayward, Alan F Wright, Ulf Gyllensten, Harry Campbell, Gerd Schmitz.

\section{Author details}

${ }^{1}$ Center for Human and Clinical Genetics, Leiden University Medical Center, S4-P, PO Box 9600, 2300, RC Leiden, Netherlands. 'Department of Clinical Genetics, DNA Diagnostics Laboratary, University of Amsterdam, Amsterdam, Netherlands. ${ }^{3}$ Genetic Epidemiology Unit, Departments of Epidemiology and Clinical Genetics, Erasmus University Medical Center, Rotterdam, Netherlands. ${ }^{4}$ Department of Physiology and Biophysics, Weill Cornell Medical College in Qatar, Education City, Qatar Foundation, PO Box 24144, Doha, State of Qatar. ${ }^{5}$ Research Unit of Molecular Epidemiology, Helmholtz Zentrum München, German Research Center for Environmental Health, Neuherberg, Germany. ${ }^{6}$ Institute of Genetic Epidemiology, Helmholtz Zentrum München, German Research Center for Environmental Health, Neuherberg, Germany. ' Institute of Experimental Genetics, Genome Analysis Center, Helmholtz Zentrum München, German Research Center for Environmental Health, Neuherberg, Germany. ${ }^{8}$ Chair of Experimental Genetics, Technische Universität München, Munich, Germany. ${ }^{9}$ Institute of Bioinformatics and Systems Biology, Helmholtz Zentrum Munchen, German Research Center for Environmental Health, Neuherberg, Germany. ${ }^{10}$ Department of Endocrinology, Leiden University Medical Center, S4-P, PO Box 9600, 2300, RC Leiden, Netherlands.

Received: 9 July 2013 Accepted: 2 December 2013

Published: 9 December 2013

\section{References}

1. Gieger C, Geistlinger L, Altmaier E, Hrabe de Angelis M, Kronenberg F, Meitinger T, Mewes HW, Wichmann HE, Weinberger KM, Adamski J, et al: Genetics meets metabolomics: a genome-wide association study of metabolite profiles in human serum. PLoS Genet 2008, 4:e1000282. 2008/12/02 edn.

2. Illig T, Gieger C, Zhai G, Romisch-Margl W, Wang-Sattler R, Prehn C, Altmaier E, Kastenmuller G, Kato BS, Mewes HW, et al: A genome-wide perspective of genetic variation in human metabolism. Nat Genet 2010, 42(2):137-141.

3. Suhre K, Shin SY, Petersen AK, Mohney RP, Meredith D, Wagele B, Altmaier E, Deloukas P, Erdmann J, Grundberg E, et al: Human metabolic individuality in biomedical and pharmaceutical research. Nature 2011 477(7362):54-60.

4. Demirkan A, van Duijn CM, Ugocsai $P$, Isaacs A, Pramstaller PP, Liebisch $G$ Wilson JF, Johansson A, Rudan I, Aulchenko YS, et al: Genome-wide association study identifies novel loci associated with circulating phospho- and sphingolipid concentrations. PLoS Genet 2012, 8(2):e1002490. 
5. Wang-Sattler R, Yu Z, Herder C, Messias AC, Floegel A, He Y, Heim K, Campillos M, Holzapfel C, Thorand B, et al: Novel biomarkers for pre-diabetes identified by metabolomics. Mol Syst Biol 2012, 8:615.

6. Xu T, Holzapfel C, Dong X, Bader E, Yu Z, Prehn C, Perstorfer K, Jaremek M, Roemisch-Margl W, Rathmann W, et al: Effects of smoking and smoking cessation on human serum metabolite profile: results from the KORA cohort study. BMC Med 2013, 11:60.

7. Suhre K, Gieger C: Genetic variation in metabolic phenotypes: study designs and applications. Nat Rev Genet 2012, 13(11):759-769.

8. Li M, Wang K, Grant SF, Hakonarson H, Li C: ATOM: a powerful gene-based association test by combining optimally weighted markers. Bioinformatics 2009, 25(4):497-503.

9. Liu JZ, McRae AF, Nyholt DR, Medland SE, Wray NR, Brown KM, Hayward NK, Montgomery GW, Visscher PM, Martin NG, et al: A versatile gene-based test for genome-wide association studies. Am J Hum Genet 2010, 87(1):139-145.

10. Wang K, Li M, Bucan M: Pathway-based approaches for analysis of genomewide association studies. Am J Hum Genet 2007, 81 (6):1278-1283.

11. Stobbe MD, Houten SM, Jansen GA, van Kampen AH, Moerland PD: Critical assessment of human metabolic pathway databases: a stepping stone for future integration. BMC Syst Biol 2011, 5:165.

12. Kanehisa M, Goto S, Furumichi M, Tanabe M, Hirakawa M: KEGG for representation and analysis of molecular networks involving diseases and drugs. Nucleic Acids Res 2010, 38(Database issue):D355-D360.

13. Romero P, Wagg J, Green ML, Kaiser D, Krummenacker M, Karp PD: Computational prediction of human metabolic pathways from the complete human genome. Genome Biol 2005, 6(1):R2.

14. Wolstencroft K, Haines R, Fellows D, Williams A, Withers D, Owen S, SoilandReyes S, Dunlop I, Nenadic A, Fisher P, et al: The taverna workflow suite: designing and executing workflows of web services on the desktop, web or in the cloud. Nucleic Acids Res 2013,41(Web Server issue):W557-W561.

15. Goble CA, Bhagat J, Aleksejevs S, Cruickshank D, Michaelides D, Newman D, Borkum M, Bechhofer S, Roos M, Li P, et al: myExperiment: a repository and social network for the sharing of bioinformatics workflows. Nucleic Acids Res 2010, 38(Web Server issue):W677-W682.

16. Li J, Ji L: Adjusting multiple testing in multilocus analyses using the eigenvalues of a correlation matrix. Heredity (Edinb) 2005, 95(3):221-227.

17. Stouffer SA, Suchman EA, DeVinney LC, Star SA, Williams RM Jr: Adjustment during army life. Princeton, NJ: Princeton University Press; 1949.

18. Moore JH, Asselbergs FW, Williams SM: Bioinformatics challenges for genome-wide association studies. Bioinformatics 2010, 26(4):445-455.

19. Mesirov JP: Computer science. Accessible reproducible research. Science 2010, 327(5964):415-416

20. Cook RJ, Lloyd RS, Wagner C: Isolation and characterization of CDNA clones for rat liver 10-formyltetrahydrofolate dehydrogenase. J Biol Chem 1991, 266(8):4965-4973.

21. Anguera MC, Field MS, Perry C, Ghandour H, Chiang EP, Selhub J, Shane B, Stover PJ: Regulation of folate-mediated one-carbon metabolism by 10-formyltetrahydrofolate dehydrogenase. J Biol Chem 2006, 281 (27):18335-18342.

22. Krebs HA, Hems $\mathrm{R}$, Tyler $\mathrm{B}$ : The regulation of folate and methionine metabolism. Biochem J 1976, 158(2):341-353.

23. Fu TF, Maras B, Barra D, Schirch V: A noncatalytic tetrahydrofolate tight binding site is on the small domain of 10-formyltetrahydrofolate dehydrogenase. Arch Biochem Biophys 1999, 367(2):161-166.

24. Krupenko SA, Oleinik NV: 10-formyltetrahydrofolate dehydrogenase, one of the major folate enzymes, is down-regulated in tumor tissues and possesses suppressor effects on cancer cells. Cell Growth Differ 2002, 13(5):227-236.

25. Kim DW, Huang T, Schirch D, Schirch V: Properties of tetrahydropteroylpentaglutamate bound to 10-formyltetrahydrofolate dehydrogenase. Biochemistry 1996, 35(49):15772-15783.

26. Lamers Y, Williamson J, Gilbert LR, Stacpoole PW, Gregory JF 3rd: Glycine turnover and decarboxylation rate quantified in healthy men and women using primed, constant infusions of [1,2-(13)C2]glycine and [(2) H3]leucine. J Nutr 2007, 137(12):2647-2652.

27. Wendel AA, Lewin TM, Coleman RA: Glycerol-3-phosphate acyltransferases: rate limiting enzymes of triacylglycerol biosynthesis. Biochim Biophys Acta 2009, 1791(6):501-506.

28. Brockmoller SF, Bucher E, Muller BM, Budczies J, Hilvo M, Griffin JL, Oresic M, Kallioniemi O, lljin K, Loibl S, et al: Integration of metabolomics and expression of glycerol-3-phosphate acyltransferase (GPAM) in breast cancer-link to patient survival, hormone receptor status, and metabolic profiling. J Proteome Res 2012, 11(2):850-860.
29. Beard RS Jr, Bearden SE: Vascular complications of cystathionine betasynthase deficiency: future directions for homocysteine-to-hydrogen sulfide research. Am J Physiol Heart Circ Physiol 2011, 300(1):H13-H26.

30. She QB, Hayakawa T, Tsuge H: Alteration in the phosphatidylcholine biosynthesis of rat liver microsomes caused by vitamin B6 deficiency. Biosci Biotechnol Biochem 1995, 59(2):163-167.

31. Namekata K, Enokido Y, Ishii I, Nagai Y, Harada T, Kimura H: Abnormal lipid metabolism in cystathionine beta-synthase-deficient mice, an animal model for hyperhomocysteinemia. J Biol Chem 2004, 279(51):52961-52969.

32. Devlin AM, Singh R, Wade RE, Innis SM, Bottiglieri T, Lentz SR: Hypermethylation of Fads2 and altered hepatic fatty acid and phospholipid metabolism in mice with hyperhomocysteinemia. J Biol Chem 2007, 282(51):37082-37090.

33. Ikeda K, Kubo A, Akahoshi N, Yamada H, Miura N, Hishiki T, Nagahata Y, Matsuura T, Suematsu M, Taguchi R, et al: Triacylglycerol/phospholipid molecular species profiling of fatty livers and regenerated non-fatty livers in cystathionine beta-synthase-deficient mice, an animal model for homocysteinemia/homocystinuria. Anal Bioanal Chem 2011, 400(7):1853-1863.

34. Wood AR, Hernandez DG, Nalls MA, Yaghootkar H, Gibbs JR, Harries LW, Chong S, Moore M, Weedon MN, Guralnik JM, et al: Allelic heterogeneity and more detailed analyses of known loci explain additional phenotypic variation and reveal complex patterns of association. Hum Mol Genet 2011, 20(20):4082-4092.

35. Hettne KM, Wolstencroft K, Belhajjame K, Goble CA, Mina E, Dharuri H, De Roure D, Verdes-Montenegro L, Garrido J, Roos M: Best practices for workflow design: how to prevent workflow decay. Proceedings of the 5th international workshop on semantic web applications and tools for life sciences. Paris, France; 2012. CEUR-WS.org Volume 952.

doi:10.1186/1471-2164-14-865

Cite this article as: Dharuri et al: Automated workflow-based exploitation of pathway databases provides new insights into genetic associations of metabolite profiles. BMC Genomics 2013 14:865.

\section{Submit your next manuscript to BioMed Central and take full advantage of:}

- Convenient online submission

- Thorough peer review

- No space constraints or color figure charges

- Immediate publication on acceptance

- Inclusion in PubMed, CAS, Scopus and Google Scholar

- Research which is freely available for redistribution

Submit your manuscript at www.biomedcentral.com/submit
C) Biomed Central 